\title{
Medidor prismático hexagonal: uma alternativa à macromedição
}

\author{
Measurement with hexagonal prismatic configuration: \\ an alternative to macromeasuring
}

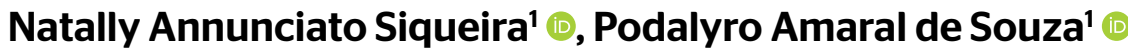

$\square$

\begin{abstract}
RESUMO
A medição de vazão é fundamental para a gestão de processos que envolvem fluidos. Em empresas de saneamento, contabilizar as entradas e saídas de água é essencial para gerir faturamentos e perdas, podendo ser mensurada por meio de variados instrumentos com diferentes princípios de aplicação. Dados os altos custos de aquisição dos macromedidores usuais, bem como as dificuldades de instalação e manutenção, a macromedição no Brasil é deficiente. O tubo Pitot Coleé um medidor capaz de determinar a vazão em tubulações pelo diferencial de pressão. Suas principais vantagens são o baixo custo e a fácil instalação, que pode ocorrer com a tubulação em carga. Em contrapartida, suas peças são frágeis e de formato complexo. Este trabalho propõe a construção de um medidor de vazão simples e robusto com tomadas de pressão instaladas em faces opostas de um prisma hexagonal e avalia a viabilidade na mediação de vazão. Os resultados mostraram-se promissores dadas a estabilidade e a forte correlação linear obtidas no coeficiente de calibração.
\end{abstract}

Palavras-chave: medidor de vazão; tubo de Pitot; medidor de velocidade.

\begin{abstract}
Flow measurements are essential for processes management involving fluids. In water companies, measuring inlets and outlets of water is essential to manage revenue and water losses. The water can be measured through diverse instruments with different application principles. Due to the high acquisition costs of the usual water flow meters, as well the difficulties of installation and maintenance, macro meter in Brazil is deficient. The Pitot Cole Tube is able to determine flow in pipes through the pressure differential. The main advantages are the low cost and easy installation, that may occur with the pipe under load. However, its peaces are fragile and complex. This paper propose the construction of a simple and robust instrument to measure the water flow. The prototype is build with pressure plugs installed on opposite faces of a hexagonal prism, then is evaluated of its effectiveness in water flow measurements. The results were promising given the stability obtained in the calibration coefficient.
\end{abstract}

Keywords: flow meter; Pitot tube; velocity meter.

\section{INTRODUÇÃO}

Dados a importância da água e o aumento das demandas hídricas em áreas urbanas, faz-se necessária uma melhor gestão e eficiência do sistema público de água. Desde a captação da água bruta até a distribuição no imóvel do consumidor, ocorrem perdas; o processo de distribuição está relacionado aos maiores índices, estimando-se que, no Brasil, no ano de 2016, as perdas de água na distribuição atingiram o valor de $36,7 \%$, e, em países com sistemas mais eficientes, não alcançaram 10\% (SNIS, 2018).

A macromedição subsidia elementos que permitem elaborar o diagnóstico operacional, a gestão de perdas de água, o controle da eficiência e a medição do faturamento, requerendo confiabilidade dos dados apresentados.
No mercado, há diferentes dispositivos para a medição de vazão. A escolha do equipamento deve analisar a necessidade da aplicação, as particularidades do escoamento e as características do medidor como incerteza, custos, tempo de vida, facilidade de instalação e viabilidade de realização da calibração.

Nesse contexto, para a medição de vazão em condutos forçados, os medidores comumente utilizados são os eletromagnéticos, os de vórtice, os de velocidade, os ultrassônicos e os de pressão diferencial.

Com o objetivo de avaliar quantitativamente o nível de medição nos sistemas de abastecimento de água, o indicador de macromedição apresenta a proporção do volume de água que foi disponibilizado para a distribuição e que foi medido. No Brasil, o valor médio do indicador é 
de 76,0\%, no entanto há disparidade entre as regiões do país. O Sudeste apresenta o indicador mais favorável, 87,3\%, enquanto a Região Norte apresenta apenas $43,5 \%$. Isto, por sua vez, indica a necessidade de melhoria na gestão de sistemas hídricos, já que a ausência de macromedidores inviabiliza a contabilização do volume de água utilizado no processo (SNIS, 2018).

Tal deficiência é consequência dos altos custos de aquisição dos macromedidores usuais, bem como das dificuldades de instalação e manutenção.

Ante a necessidade de aumentar o nível de medição em sistemas públicos de abastecimento, este trabalho propõe a aplicação de um medidor de vazão cujo princípio de funcionamento assemelha-se ao tubo Pitot Cole, contudo as tomadas de pressão estão instaladas em faces opostas de um prisma hexagonal, o que deixa o equipamento simples e robusto.

\section{METODOLOGIA}

A construção do protótipo do medidor, da bancada para os ensaios e a sequência dos ensaios para calibração estão descritas a seguir.

\section{O protótipo}

O elemento primário do protótipo é constituído de um tubo prismático hexagonal com diâmetro de $14,5 \mathrm{~mm}$, posicionado transversalmente entre duas flanges com diâmetro nominal de $150 \mathrm{~mm}$. Na posição central do prisma hexagonal, posicionadas em faces opostas, estão as duas tomadas de pressão. Trata-se de orifícios que possuem a função de comunicar, por meio de tubos de cobre, as pressões pontuais. A Figura 1 ilustra o protótipo ensaiado.

\section{A bancada de ensaio}

O protótipo foi instalado em uma bancada de testes, em um trecho reto de uma tubulação de $156 \mathrm{~mm}$ de diâmetro interno.

A jusante da instalação, encontra-se uma válvula gaveta, responsável pelo controle da vazão, de acordo com a graduação de sua abertura,

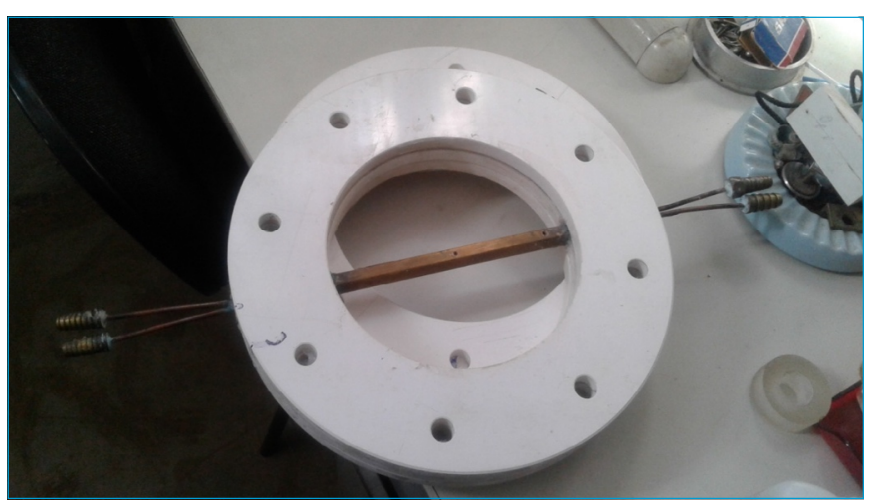

Figura 1 - Protótipo. e um vertedor triangular com capacidade de 50 L.s ${ }^{-1}$, responsável pela aferição do protótipo.

A montante do medidor, encontra-se uma válvula gaveta, com a função de bloqueio, e um reservatório de nível constante situado a uma cota de 8,0 m em relação do eixo do tubo.

\section{O ensaio}

A variação da vazão deu-se por meio de graduações no registro de gaveta instalado a jusante do protótipo e a montante do vertedor. Para cada vazão, realizaram-se leituras da lâmina de água no vertedor triangular assim como leituras manométricas.

Para aumentar o diferencial de leitura de pressão e assim diminuir o erro nas leituras, inclinou-se o quadro de manômetros e anotou-se o ângulo de inclinação.

\section{RESULTADOS E DISCUSSÃO}

\section{Equacionamentos}

O equacionamento básico para o protótipo assemelha-se ao equacionamento utilizado para o tubo Pitot Cole em que é aplicado Bernoulli a ambas tomadas de pressão. Das deduções, obtém-se que a velocidade (v) é função de um coeficiente de ajuste (k) que depende da calibração do equipamento, da aceleração da gravidade (g) e do diferencial de pressão $(\Delta \mathrm{h})$, conforme Equação 1.

$\mathrm{v}=\mathrm{k} \sqrt{2 \mathrm{~g} \Delta \mathrm{h}}$

Posteriormente, a vazão (Q) é determinada pela Equação 2, da continuidade que depende da velocidade média do fluido $(\overline{\mathrm{u}})$ e da área da seção transversal (A).

$\mathrm{Q}=\overline{\mathrm{u}} \cdot \mathrm{A}$

Como o perfil de velocidades dentro das tubulações produz velocidade nula na parede do tubo e máxima no centro, tem-se que, para a calibração do protótipo, é necessário encontrar a relação entre a velocidade média, obtida pelo vertedor, e a velocidade máxima, dada pelo protótipo.

Visando modelar o perfil de velocidades e fazendo uso dos conceitos da entropia máxima, Chiu, Li e Lu (1993) propuseram uma equação dependente apenas do parâmetro de entropia e da velocidade máxima (Equação 3).
$\frac{\overline{\mathrm{u}}}{\mathrm{u}_{\operatorname{máx}}}=\frac{\mathrm{e}^{\mathrm{M}}}{\mathrm{e}^{\mathrm{M}}-1}-\frac{1}{\mathrm{M}}$
Em que: 
$\overline{\mathrm{u}}=$ a velocidade média;

$\mathrm{u}_{\text {máx }}=$ a velocidade máxima;

$\mathrm{M}=\mathrm{o}$ parâmetro de entropia.

Relacionando-se o número de Reynolds ao aplicar as velocidades média $(\mathrm{Re})$ e máxima $\left(\mathrm{Re}_{\mathrm{maxx}}\right)$, tem-se a Equação 4 :

$\frac{\operatorname{Re}}{\operatorname{Re}_{\text {máx }}}=\frac{\overline{\mathrm{u}}}{\mathrm{u}_{\text {máx }}}$

Seguindo os conceitos da entropia máxima, tem-se a seguinte relação entre Re e M (Equação 5):

$\frac{\mathrm{Re}^{0,9995}}{422,377}=\mathrm{e}^{\mathrm{M}}-1$

O uso das Equações 3, 4 e 5 permite a obtenção de valores de $\mathrm{Re}_{\text {máx }}$ associados a Re. O ajuste desses valores é a Equação 6, com $\mathrm{r}^{2}=0,9999$.

$\ln \operatorname{Re}=-0,0036\left(\ln \operatorname{Re}_{\text {máx }}\right)^{2}+1,1267\left(\ln \operatorname{Re}_{\text {máx }}\right)-1,1931$

\section{Ensaio de calibração}

Das medições realizadas com o vertedor triangular, as vazões ensaiadas variaram de $3,56 \times 10^{-3} \mathrm{~m}^{3} \cdot \mathrm{s}^{-1}$ a $22,79 \times 10^{-3} \mathrm{~m}^{3} \cdot \mathrm{s}^{-1}$, ou velocidades médias variando entre 0,19 e $1,20 \mathrm{~m} \cdot \mathrm{s}^{-1}$.

O ensaio não ocorreu com velocidades superiores dados os elevados números de conexões presentes na instalação dos manômetros que permitiam a entrada de ar de difícil detecção.

Tendo os valores da vazão, primeiramente determinaram-se os números de Reynolds (Equação 7):

$R e=\frac{Q_{V^{*}} D}{v \cdot A}$

Em que:

$\mathrm{D}=\mathrm{o}$ diâmetro do tubo;

$\mathrm{Q}_{\mathrm{v}}=\mathrm{a}$ vazão medida pelo vertedor;

$\mathrm{v}=\mathrm{a}$ viscosidade cinemática.

Com os valores de Re substituídos na Equação 6, obtiveram-se os correspondentes valores de $\operatorname{Re}_{\text {máx }}$ o que propiciou a obtenção dos valores de $\mathrm{u}_{\text {máx }}$, pela Equação 8 .

$\mathrm{u}_{\text {máx }}=\frac{\mathrm{Re}_{\text {máx }} \cdot v}{\mathrm{D}}$

O último passo é aplicar a Equação 1 utilizando-se o valor do diferencial de pressão conseguido na leitura com o protótipo e, assim, obter o coeficiente de calibração.
A Tabela 1 apresenta os dados alcançados por meio do ensaio de calibração: a vazão obtida pelo vertedor triangular, o diferencial de pressão inserido pelo protótipo, o número de Reynolds para vazão média e máxima, a velocidade máxima e o coeficiente de calibração calculado para cada ensaio.

Na Figura 2, estão plotados os valores de $(2 \mathrm{~g} \Delta \mathrm{h})^{0,5}$ e $\mathrm{u}_{\text {máx }}$ obtidos do ensaio de calibração. $\mathrm{O}$ ajuste desses pares de valores pelo método dos mínimos quadrados forneceu a Equação 9, de aferição do dispositivo como medidor da vazão máxima, com índice de determinação $r^{2}=0,9994$.

$\mathrm{u}_{\text {máx }}=0,796 \sqrt{2 g \Delta h}$

\section{Algoritmo para a obtenção da vazão}

Descreve-se o algoritmo para determinar a vazão utilizando-se o protótipo:

- $\quad$ medir o diferencial de pressão $(\Delta \mathrm{h})$;

- calcular a velocidade máxima $\left(\mathrm{u}_{\text {máx }}\right)$ conforme a Equação 9;

- calcular o número de Reynolds para a velocidade máxima $\left(\mathrm{Re}_{\text {máx }}\right)$ seguindo a Equação 8;

- calcular o número de Reynolds (Re) conforme a Equação 6;

- calcular a velocidade média $(\overline{\mathrm{u}})$ conforme a Equação 10:

$\overline{\mathrm{u}}=\frac{\mathrm{Re} \cdot \mathrm{v}}{\mathrm{D}}$

- calcular a vazão (Q) conforme a Equação 2.

\section{CONCLUSÕES}

O medidor de vazão proposto, baseado no princípio de funcionamento de um tubo Pitot Cole, construído sobre um prisma de seção hexagonal, apresentou resultados bastante satisfatórios.

Essa afirmação está corroborada pelo comportamento linear observado pela lei de aferição do medidor (Figura 2), com ajuste linear com índice de determinação $\mathrm{r}^{2}=0,9994$.

Pode-se justificar o valor constante do coeficiente de velocidade, mesmo para pequenas vazões, dado o formato do medidor que garante o descolamento nas arestas inferior e superior e escoamento turbulento a jusante do medidor.

Uma vez aferido o medidor, a obtenção da vazão pode ser feita pelo algoritmo expresso neste trabalho.

Mesmo com os bons resultados apresentados por este novo medidor, alerta-se que o valor encontrado para o coeficiente de calibração restringe-se à relação entre o diâmetro externo da seção hexagonal e o diâmetro do tubo $(\mathrm{d} / \mathrm{D}=0,094)$. Sendo assim, recomenda-se que outros valores de $\mathrm{d} / \mathrm{D}$ sejam ensaiados em futuras pesquisas para se determinar a dependência do coeficiente de velocidade e d/D. 
Tabela 1 - Medições e resultados do ensaio de calibração.

\begin{tabular}{|c|c|c|c|c|c|}
\hline $\operatorname{Vazão}\left(m^{3} \cdot s^{-1}\right)$ & Diferencial de pressão (m) & $\begin{array}{l}\text { Reynolds - velocidade } \\
\text { média }\end{array}$ & $\begin{array}{l}\text { Reynolds - velocidade } \\
\text { máxima }\end{array}$ & Velocidade máxima $\left(\mathrm{m}^{\mathrm{s}} \mathrm{s}^{-1}\right)$ & Coeficiente de calibração \\
\hline 0,0036 & 0,006 & 29.032 & 37.573 & 0,241 & 0,733 \\
\hline 0,0042 & 0,008 & 34.579 & 44.380 & 0,284 & 0,742 \\
\hline 0,0047 & 0,009 & 38.510 & 49.176 & 0,315 & 0,772 \\
\hline 0,0060 & 0,014 & 48.872 & 61.729 & 0,396 & 0,755 \\
\hline 0,0063 & 0,015 & 51.303 & 64.658 & 0,414 & 0,767 \\
\hline 0,0069 & 0,018 & 56.142 & 70.473 & 0,452 & 0,760 \\
\hline 0,0086 & 0,029 & 69.809 & 86.801 & 0,556 & 0,744 \\
\hline 0,0088 & 0,030 & 72.124 & 89.555 & 0,574 & 0,747 \\
\hline 0,0094 & 0,035 & 77.035 & 95.385 & 0,611 & 0,743 \\
\hline 0,0104 & 0,041 & 84.541 & 104.270 & 0,668 & 0,745 \\
\hline 0,0127 & 0,061 & 103.881 & 127.046 & 0,814 & 0,745 \\
\hline 0,0140 & 0,072 & 114.460 & 139.441 & 0,894 & 0,752 \\
\hline 0,0143 & 0,073 & 116.833 & 142.216 & 0,912 & 0,761 \\
\hline 0,0146 & 0,078 & 119.422 & 145.242 & 0,931 & 0,753 \\
\hline 0,0153 & 0,087 & 124.893 & 151.628 & 0,972 & 0,744 \\
\hline 0,0154 & 0,085 & 125.852 & 152.746 & 0,979 & 0,759 \\
\hline 0,0159 & 0,092 & 129.338 & 156.809 & 1,005 & 0,748 \\
\hline 0,0189 & 0,131 & 154.491 & 186.031 & 1,193 & 0,743 \\
\hline 0,0212 & 0,163 & 172.692 & 207.081 & 1,327 & 0,744 \\
\hline
\end{tabular}

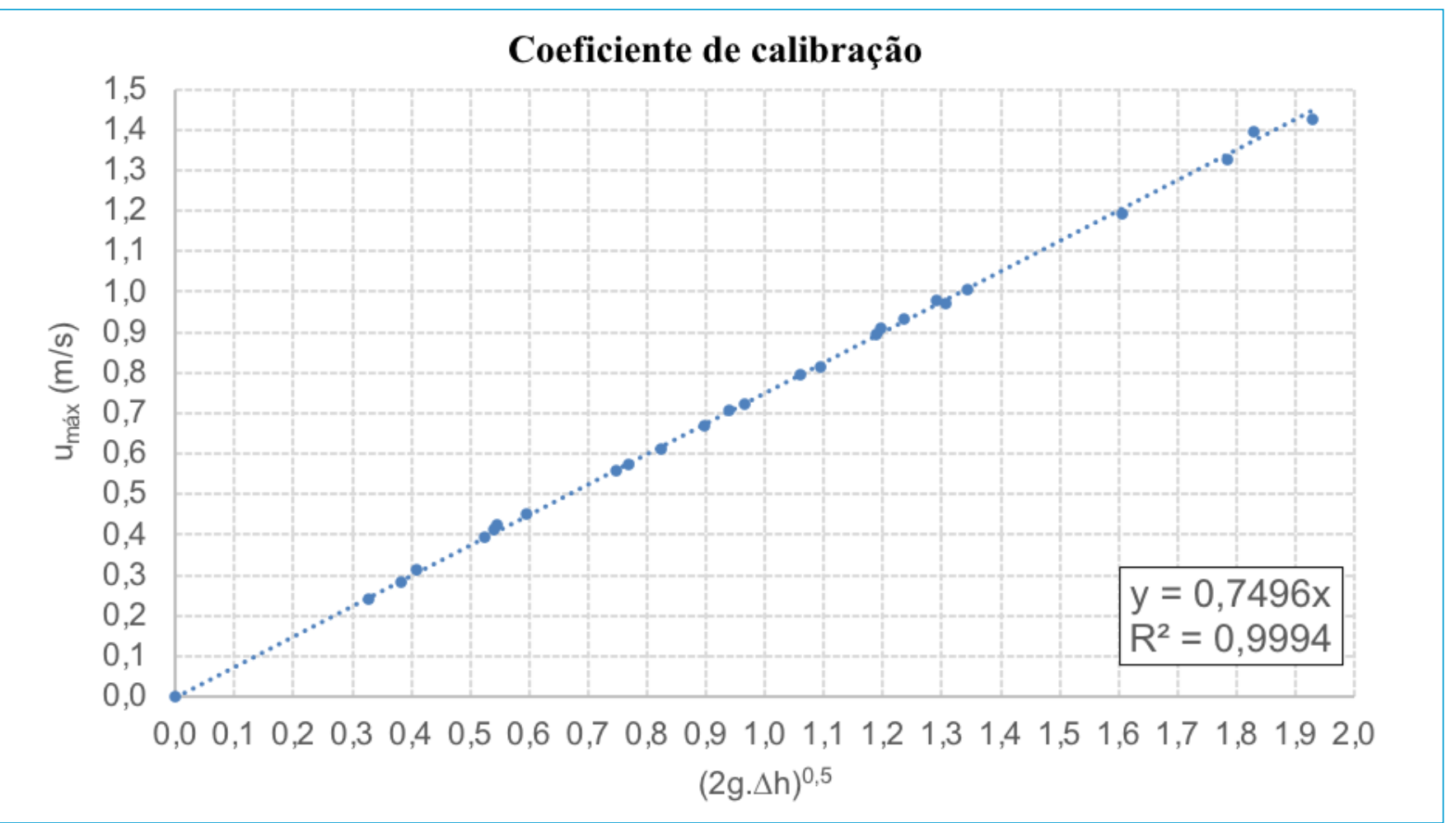

Figura 2 - Determinação do coeficiente de calibração. 


\section{REFERENCIAS}

CHIU, C.; LI, G.-F.; LU, J.-M. Application of probability and entropy concepts in pipe flow study. Journal of Hydraulic Engineering, Reston, v. 119, n. 6, p. 742-756, 1993. https://doi.org/10.1061/ (ASCE)0733-9429(1993)119:6(742)
SISTEMA NACIONAL DE INFORMAÇÃO SOBRE SANEAMENTO (SNIS). Diagnóstico dos Serviços de Esgotos. SNIS, 2018. Disponível em: <http://www.snis.gov.br/diagnostico-agua-e-esgotos/diagnosticoae-2016>. Acesso em: 18 fev. 2018. 